\title{
EFEITO DO ALUMÍNIO NO DESENVOLVIMENTO E NUTRIÇÃO DE Tapirira guianensis AUBLET EM SOLUÇÃO HIDROPÔNICA
}

\author{
Ricardo Miranda de Britez ${ }^{1}$ \\ Carlos Bruno Reissmann ${ }^{2}$ \\ Toshihiro Watanabe \\ Mitsuru Osaki ${ }^{3}$
}

\begin{abstract}
RESUMO
Objetivando-se testar Tapirira guianensis, espécie de ampla distribuição na América do Sul, frente a níveis crescentes de $\mathrm{Al}(0,0,5 \mathrm{mM}, 1,0 \mathrm{mM}, 2,0 \mathrm{mM}, 4,0 \mathrm{mM}$ e $6,0 \mathrm{mM})$, foi realizado experimento utilizando-se solução hidropônica como substrato. Houve um efeito benéfico do Al, apresentando um aumento do crescimento de diferentes partes da planta a medida que aumentaram as quantidades de Al na solução. Ocorreu uma diminuição dos teores de nutrientes na planta e um aumento do Al. Na raiz o teor de Al aumentou cerca de 50 vezes no tratamento com maior concetração de $\mathrm{Al}$ em relação a testemunha. Considerando-se o conteúdo de nutrientes observou-se uma tendência de aumento para todos os macronutrientes em todas as partes da planta, com exceção do $\mathrm{P}$, que teve seu conteúdo diminuído. Conclui-se que Tapirira guianensis é altamente tolerante ao Al, desenvolvendo mecanismos que inibem sua toxidez. Destes possivelmente alguns estão relacionados a excreção de substâncias orgânicas que quelam o Al tornando-o menos tóxico no meio, e o outro, refere-se ao acumulo na raiz, não permitindo que este seja transportado para a parte aérea em quantidades que possam tornálo tóxico.

Palavras chave: toxidez de Alumínio, Alumínio em árvores tropicais, nutrição mineral, exsudados da raiz
\end{abstract}

\section{EFFECTS OF ALUMINUM ON GROWTH AND NUTRITION OF Tapirira guianensis AUBLET IN A HIDROPONIC CULTURE}

\begin{abstract}
In this study, the main purpose is to test Tapirira guianensis, a tree species with a wide dispersion range in South America to increasing levels of $\mathrm{Al}(0,0,5 \mathrm{mM}, 1,0 \mathrm{mM}, 2,0 \mathrm{mM}, 4,0 \mathrm{mM}$ and $6,0 \mathrm{mM})$. The experiment was developed in a greenhouse utilizing hydroponic solution as substrate. The seedlings showed an increase in biomass production with the $\mathrm{Al}$ rates increased. The levels of nutrients in the plant decrease with exception of $\mathrm{Al}$. The concentration of $\mathrm{Al}$ in the roots was fifty times higher in the tested treatments than in the control. In all parts of plant the amount of absorbed nutrients increased, mainly the macronutrients, with exception for P. It was concluded that Tapirira guianensis is highly $\mathrm{Al}$ tolerant by developing mechanisms avoiding $\mathrm{Al}$ toxicity. This mechanisms was related to exudation of organic substances with the chelation of the Al changed its toxicity in the medium, and the $\mathrm{Al}$ accumulation in the roots not permitting the transport to the leaves.

Key-words: Aluminum toxicity, Aluminum in tropical trees, mineral nutrition, root exudates
\end{abstract}

\section{INTRODUÇÃO}

O alumínio é o metal mais abundante e o terceiro elemento em quantidade na crosta terrestre. A toxidez do Al é reconhecida desde o inicio do século como um importante fator limitante no crescimento das plantas em muitos solos ácidos, sendo mais severa em solos com pH abaixo de 5.0 (Foy, 1975; Foy et al. 1978). As formas de $\mathrm{Al}$ tóxicas às plantas suscitam muito debate. Em condições ácidas o $\mathrm{Al}^{+3}$ reage com outros ligantes formando várias espécies químicas, uma ou mais destas é rizotóxica, ou seja, inibe o crescimento da raiz (Kinraide, 1991).

\footnotetext{
1 rmbritez@netpar.com.br, Dr, - SPVS; - Rua Dr. Eneas Marques dos Santos nº 17, 80740-440, Curitiba, Paraná

reissman@agrarias.UFPR.br; Prof. Dept. de Solos da UFPR. - Rua dos Funcionários 1540, 80035-050, Curitiba, Paraná.

3 nabe@chem.agr.hokudai.ac.jp. Dr Agriculture, Hokkaido University; End. - Kiat-9, Nishi-9, Kitaku, Sapporo 060-8589, Japan
} 
Os solos ácidos desenvolvem-se preferencialmente em clima úmido. A água é um importante fator de formação desses solos, pois remove vagarosamente os minerais mais solúveis. A sílica tem um papel importante neste processo, quando persiste no solo, não sendo lixiviada, fornam-se os minerais de argila 2:1, apresentando uma baixa solubilidade de Al. Mas quando é removida (alta pluviosidade), formam-se os minerais de argila 1:1, aumentando a solubilidade do $\mathrm{Al}$ (Lindsay e Walthall, 1996).

Eswaran et al. (1997), estimaram que $26 \%$ das terras do planeta $(37,77$ milhões de $\mathrm{Km}^{2}$ ) que não estão totalmente ocupadas pelo gelo são constituídas por solos ácidos, e em 20 $\%$ destas terras a acidez atinge também o subsolo (profundidade entre 25 e $75 \mathrm{~cm}$ ). A América do Sul possui a maior área, 11,8 milhões $\mathrm{Km}^{2}$, representando $66,1 \%$ dos solos ácidos, sendo no Brasil a maior ocorrência.

Em uma escala global existem dois cinturões principais de solos ácidos: um na zona temperada úmida norte coberta principalmente por florestas de coníferas; e outra no trópico úmido, coberto por savanas e florestas tropicais, com os respectivos solos característicos destas zonas (Uexküll e Mutert, 1995). Portanto o mesmo clima úmido que propicia o desenvolvimento das florestas, é também o que origina os solos ácidos com a conseqüente toxidez do Al.

Estudos em relação à toxidez do $\mathrm{Al}$ datam do início do século principalmente em culturas agrícolas e só recentemente a avaliação de toxidez de $\mathrm{Al}$ em árvores tem se intensificado principalmente em função do efeito das chuvas ácidas na Europa, Rússia e América do Norte, promovendo a poluição das águas e diminuição da produtividade das florestas (Joslin e Wolfe, 1989; Sposito, 1996).

Os mecanismos de adaptação à toxidez do $\mathrm{Al}$ em espécies das florestas tropicais ainda são desconhecidos. Este trabalho, tem como objetivo testar uma espécie arbórea (Tapirira guianensis), frente a níveis crescentes de Al, utilizando solução hidropônica como substrato.

\section{METODOLOGIA}

A espécie escolhida para este experimento é de ampla ocorrência na América do Sul, sendo uma das espécies dominantes nas florestas da planície litorânea da Ilha do Mel, adaptada a solos de extrema acidez e baixa fertilidade (Britez et al.1997). O experimento foi realizado em casa de vegetação sem controle de temperatura, no Setor de Ciências Agrárias da Universidade Federal do Paraná em Curitiba, utilizando-se 12 caixas de 70 litros com solução nutritiva, devidamente oxigenada. As plântulas de $T$. guianensis foram obtidas pela coleta de plântulas de regeneração natural. No laboratório, as raízes foram lavadas com água deionizada e hipoclorito de sódio. Em cada caixa foram conduzidas 10 plantas, permanecendo em solução nutritiva durante um período de dois meses para adaptação. $\mathrm{O}$ $\mathrm{pH}$ da solução foi mantido em 4,0. Posteriormente foram aplicados os seguintes tratamentos : testemunha, $0,5 \mathrm{mM}, 1,0 \mathrm{mM}$, 2,0 mM, $4 \mathrm{mM}$ e $6 \mathrm{mM}$ de Al sob a forma de $\mathrm{AlCl}_{3} 6 \mathrm{H}_{2} \mathrm{O}$. A solução nutritiva utilizada foi baseada no trabalho de Osaki et al. (1997), e, apresentava a seguinte composição: $2,14 \mathrm{mM}$ $\mathrm{N}\left(\left(\mathrm{NH}_{4}\right)_{2} \mathrm{SO}_{4}\right), 0,32 \mathrm{mM} \mathrm{P}\left(\mathrm{NaH}_{2} \mathrm{PO}_{4} \cdot 2 \mathrm{H}_{2} \mathrm{O}\right)$, $0,77 \mathrm{mM} \mathrm{K}\left(\mathrm{K}_{2} \mathrm{SO}_{4}: \mathrm{KCL}=1: 1\right), 1,25 \mathrm{mM} \mathrm{Ca}$ $\left(\mathrm{CaCl}_{2} \cdot 2 \mathrm{H}_{2} \mathrm{O}\right), 0,82 \mathrm{mM} \mathrm{Mg}\left(\mathrm{MgSO}_{4} \cdot 7 \mathrm{H}_{2} \mathrm{O}\right)$, $35,8 \mu \mathrm{M} F e\left(\mathrm{FeSO}_{4} .7 \mathrm{H}_{2} \mathrm{O}\right), 9,1 \mu \mathrm{M} \quad \mathrm{Mn}$ $\left(\mathrm{MnSO}_{4} \cdot 4 \mathrm{H}_{2} \mathrm{O}\right), 46,3 \mu \mathrm{M} \mathrm{B}\left(\mathrm{H}_{3} \mathrm{BO}_{3}\right), 3,1 \mu \mathrm{M}$ $\mathrm{Zn} \quad\left(\mathrm{ZnSO}_{4} \cdot 7 \mathrm{H}_{2} \mathrm{O}\right), \quad 0,16 \mu \mathrm{M} \quad \mathrm{Cu}$ $\left(\mathrm{CuSO}_{4} \cdot 5 \mathrm{H}_{2} \mathrm{O}\right), \quad 0,05 \quad \mu \mathrm{M} \quad \mathrm{Mo}$ $\left(\left(\mathrm{NH}_{4}\right)_{6} \cdot \mathrm{Mo}_{7} \mathrm{O}_{24} \cdot 4 \mathrm{H}_{2} \mathrm{O}\right)$.

Os tratamentos foram aplicados no período entre 06/99 e 12/99, os parâmetros analisados foram medidos pelo agrupamento de 3 plantas por caixa. Em relação aos parâmetros biométricos da planta foram avaliadas: o comprimento das raízes, altura da planta, diâmetro do caule, número de folhas, peso seco das folhas, caule, raízes e relação número de folhas/peso seco das folhas. $\mathrm{O}$ comprimento das raízes foi medido pelo método da linha interceptada (Tennant, 1975). A altura da planta foi medida através de régua. O diâmetro do caule na altura do colo, mediuse com paquímetro.

Foram analisados $\mathrm{N}, \mathrm{P}, \mathrm{K}, \mathrm{Ca}, \mathrm{Mg}$, $\mathrm{Fe}, \mathrm{Mn}, \mathrm{Cu}, \mathrm{Zn}$ e $\mathrm{Al}$ nas folhas, caule e raízes, no Laboratório de Nutrição de Plantas da Universidade Federal do Paraná, Setor de Ciências Agrárias, em Curitiba, Estado do Paraná. O material vegetal, depois de seco a temperatura de $70^{\circ} \mathrm{C}$ foi homogeneizado e 
moído. O preparo e digestão das amostras foram realizados pelo método de via seca, de acordo com as instruções de Hildebrand et al. (1976/77).

O P foi determinado pelo método colorimétrico com vanato-molibdato de amônio (cor amarela), em espectrofotômetro UV/VIS- 554 Perkin-Elmer. Os demais elementos em espectrofotômetro de absorção atômica 2380 Perkin-Elmer. $\quad \mathrm{O} \quad \mathrm{N}$ foi determinado pelo método de Kjeldahl.

$\mathrm{Na}$ solução hidropônica foram analisados o Al total, Al monomérico e $\mathrm{Al}$ orgânico. $\mathrm{O} \mathrm{Al}$ monomérico foi medido pela metodologia do aluminon através de método colorimétrico segundo Kerven et al. (1989). Para avaliação do Al complexado com substâncias orgânicas na solução hidropônica, mediu-se o $\mathrm{Al}$ em espectrofotômetro de absorção atômica, antes, e após a incineração e solubilização com $\mathrm{HCl}$, adaptando-se o método sugerido por Bloom e Erich (1996), utilizado para destruir complexos orgânicos em medidas de $\mathrm{Al}$ total realizadas em análises de água. Este visa promover a queima da matéria orgânica, que interfere na análise do $\mathrm{Al}$, a diferença entre as duas medidas, estimou o $\mathrm{Al}$ complexado com substâncias orgânicas.

$\mathrm{O}$ delineamento utilizado foi $\mathrm{o}$ de blocos casualizados. A homogeneidade das variâncias foi testada pelo teste de Bartlett, sendo os valores transformados quando necessário para posterior análise de variância. Os resultados obtidos em função dos tratamentos com doses crescentes de $\mathrm{Al}$, foram avaliados através de ajuste linear e quadrático de curvas de regressão.

Para facilitar a interpretação dos dados, reuniu-se variáveis altamente relacionadas em um número menor de variáveis (fatores), através da análise fatorial do método estatístico multivariado (Johnson e Wichern, 1988). Os pesos fatoriais foram estimados pelo método de componentes principais, a escolha do número de fatores foi baseada no método de Kaiser (autovalores > 1), em algumas situações foram incluídos fatores com autovalores próximos a 1. Após a definição dos fatores, para uma melhor visualização dos dados foi realizada a rotação varimax normalizada.

\section{RESULTADOS E DISCUSSÃO}

\section{Desenvolvimento das plantas}

Em relação ao desenvolvimento das plantas, a análise de variância registrou diferenças significativas entre os tratamentos, para os parâmetros peso de folhas, caule e raiz, diâmetro do caule e a relação entre peso e número de folhas (tab. 1).

Não foram observados sintomas visuais de deficiência nutricional e todos os parâmetros biométricos tiverem tendências a aumentar frente aos diferentes tratamentos com o $\mathrm{Al}$ (tab. 2), visualizados no ajustes de curvas de regressão na figura 1 . As diferentes formas de Al na solução (tab. 3) foram positiva e altamente correlacionadas, com todos os parâmetros de desenvolvimento da planta, exceto para comprimento da raiz, ou seja, aumento no desenvolvimento das plantas à medida que cresciam as quantidades de $\mathrm{Al}$ na solução.

A tolerância das espécies e cultivares a diferentes quantidades de Al na solução é bastante variada (Wheeler et al., 1993). Em plantas sensíveis ao Al utilizadas na agricultura concentrações de até $20 \mu \mathrm{M}$ em solução nutritiva apresentam sintomas de toxidez.

As árvores são consideradas relativamente mais tolerantes. Plântulas de Betula pendula, apresentaram decréscimo no seu crescimento somente a partir de $3 \mathrm{mM}$ na solução (Goransson e Eldhuset, 1987). Na literatura relata-se resultados diferentes para uma mesma espécie, em função de diferenças na composição química da solução. Uma das razões está relacionada à modificação da atividade do $\mathrm{Al}$ na solução. A hidrólise do $\mathrm{Al}$ permite a formação de diferentes espécies, dentre elas as espécies de Al monomérico, polimérico e $\mathrm{Al}$ orgânico. Esta última não é considera tóxica. Em solução, $\mathrm{o} \quad \mathrm{Al}$ monomérico é formado pela soma do $\mathrm{Al}^{3+} \mathrm{com}$ $\mathrm{Al}(\mathrm{OH})^{2+}, \mathrm{Al}(\mathrm{OH})_{2}{ }^{+}, \mathrm{Al}(\mathrm{OH})_{3}^{0} \mathrm{e} \mathrm{AlSO}_{4}^{+}$. As quantidades de cada uma destas espécies e sua toxidez, varia conforme o $\mathrm{pH}$ e a força ionica, sendo difícil avaliar qual a forma mais tóxica devido a colinearidade (Kinraide, 1991). Pavan e Binghan (1982), em solução nutritiva mantida em $\mathrm{pH}$ 4,0, associou a diminuição do crescimento da raiz do café ao $\mathrm{Al}^{3+}$. Alva et al. (1986), em solução nutritiva com $\mathrm{pH}$ variando 
de 4 a 5 , constatou como espécies mais tóxicas para a soja, $\mathrm{Al}(\mathrm{OH})^{2+} \mathrm{e} \mathrm{Al}(\mathrm{OH})_{2}{ }^{+}$, em $\mathrm{pH} 4,5$, sendo que o $\mathrm{Al}^{3+}$ teve pouca influência no crescimento da raiz. Desta forma, soluções com pH 4,0 seriam menos tóxicas, devido as baixas concentrações destas formas mais tóxicas (Moore, citado por Mengel e Kirkby, 1987, p. 58). Segundo Kinraide (1991), o efeito verdadeiro destas espécies no crescimento da raiz ainda é obscuro.

Tabela 1: Análise de variância para os parâmetros de desenvolvimento de Tapirira guianensis, no experimento realizado em hidroponia frente a níveis crescentes de $\mathrm{Al}$

Table 1: Variance analysis for the development parameters of Tapirira guianensis, in the experiment held in hydroponic solution to increasing levels of Al

\begin{tabular}{|c|c|c|c|c|c|c|}
\hline Folhas & Graus de & Soma dos & Quadrado & & \multicolumn{2}{|c|}{ F Requerido } \\
\hline Fonte de variação & Liberdade & Quadrados & Médio & $\mathrm{F}$ & $5 \%$ & $1 \%$ \\
\hline Tratamentos & 5 & 8,90 & 1,78 & 3,49 & 2,57 & 4,11 \\
\hline Erro experimental & 27 & 13,79 & 0,51 & & & \\
\hline Total & 32 & 22,69 & & & & \\
\hline Caule & Graus de & Soma dos & Quadrado & & \multicolumn{2}{|c|}{ F Requerido } \\
\hline Fonte de variação & Liberdade & Quadrados & Médio & $\mathrm{F}$ & $5 \%$ & $1 \%$ \\
\hline Tratamentos & 5 & 2,56 & 0,513 & 5,38 & 2,57 & 4,11 \\
\hline Erro experimental & 27 & 2,57 & 0,095 & & & \\
\hline Total & 32 & 5,13 & & & & \\
\hline Raiz (peso) & Graus de & Soma dos & Quadrado & & \multicolumn{2}{|c|}{ F Requerido } \\
\hline Fonte de variação & Liberdade & Quadrados & Médio & $\mathrm{F}$ & $5 \%$ & $1 \%$ \\
\hline Tratamentos & 5 & 3,708 & 0,742 & 4,526 & 2,57 & 4,11 \\
\hline Erro experimental & 27 & 4,423 & 0,164 & & & \\
\hline Total & 32 & 8,131 & & & & \\
\hline Altura & Graus de & Soma dos & Quadrado & & \multicolumn{2}{|c|}{ F Requerido } \\
\hline Fonte de variação & Liberdade & Quadrados & Médio & $\mathrm{F}$ & $5 \%$ & $1 \%$ \\
\hline Tratamentos & 5 & 131,42 & 26,28 & 2,22 & 2,57 & 4,11 \\
\hline Erro experimental & 27 & 319,43 & 11,83 & & & \\
\hline Total & 32 & 450,86 & & & & \\
\hline Diâmetro & Graus de & Soma dos & Quadrado & & \multicolumn{2}{|c|}{ F Requerido } \\
\hline Fonte de variação & Liberdade & Quadrados & Médio & $\mathrm{F}$ & $5 \%$ & $1 \%$ \\
\hline Tratamentos & 5 & 0,026 & 0,0052 & 3,508 & 2,57 & 4,11 \\
\hline Erro experimental & 27 & 0,040 & 0,0015 & & & \\
\hline Total & 32 & 0,067 & & & & \\
\hline Raiz (comprimento) & Graus de & Soma dos & Quadrado & & \multicolumn{2}{|c|}{ F Requerido } \\
\hline Fonte de variação & Liberdade & Quadrados & Médio & $\mathrm{F}$ & $5 \%$ & $1 \%$ \\
\hline Tratamentos & 5 & 13611,9 & 2722,4 & 0,71 & 2,57 & 4,11 \\
\hline Erro experimental & 27 & 104017,2 & 3852,5 & & & \\
\hline Total & 32 & 117629,1 & & & & \\
\hline Peso/n ${ }^{0}$ folhas & Graus de & Soma dos & Quadrado & & \multicolumn{2}{|c|}{ F Requerido } \\
\hline Fonte de variação & Liberdade & Quadrados & Médio & $\mathrm{F}$ & $5 \%$ & $1 \%$ \\
\hline Tratamentos & 5 & 0,0174 & 0,0035 & 4,91 & 2,57 & 4,11 \\
\hline Erro experimental & 27 & 0,0192 & 0,0007 & & & \\
\hline Total & 32 & 0,0366 & & & & \\
\hline
\end{tabular}

ns não significativo.

* significativo a $5 \%$ de probabilidade.

** significativo a $1 \%$ de probabilidade 
Tabela 2: Médias e desvio padrão para os parâmetros de crescimento de Tapirira guianensis

Table 2: $\quad$ Average and standard deviation for the growth parameters of Tapirira guianensis

\begin{tabular}{|c|c|c|c|c|c|c|c|c|c|c|}
\hline \multirow{2}{*}{$\begin{array}{l}\text { TRATAM. } \\
\text { mM de Al }\end{array}$} & \multicolumn{3}{|c|}{ Peso Folhas (g) } & \multicolumn{2}{|c|}{ Peso Caule (g) } & \multicolumn{3}{|c|}{ Peso Raiz (g) } & \multicolumn{2}{|c|}{ Altura $(\mathrm{cm})$} \\
\hline & \begin{tabular}{l|l} 
Média & I \\
\end{tabular} & \multicolumn{2}{|c|}{ Desvio.padrão } & Média & Desvio.padrão & Média & \multicolumn{2}{|c|}{ Desvio.padrão } & Média & Desvio.padrão \\
\hline 0 & \multicolumn{2}{|l|}{2,262} & 0,465 & 0,790 & 0,200 & 0,780 & & 0,053 & 17,778 & 1,446 \\
\hline 0,5 & \multicolumn{2}{|l|}{2,053} & 0,582 & 0,795 & 0,302 & 0,858 & & 0,432 & 18,485 & 4,009 \\
\hline 1,0 & \multicolumn{2}{|l|}{2,732} & 0,808 & 0,968 & 0,319 & 1,128 & & 0,424 & 18,508 & 1,776 \\
\hline 2,0 & \multicolumn{2}{|l|}{3,045} & 0,989 & 1,153 & 0,450 & 1,232 & & 0,606 & 22,542 & 6,052 \\
\hline 4,0 & \multicolumn{2}{|l|}{3,117} & 0,588 & 1,222 & 0,254 & 1,397 & & 0,223 & 21,950 & 2,086 \\
\hline 6,0 & \multicolumn{2}{|l|}{3,685} & 0,671 & 1,678 & 0,195 & 1,883 & & 0,407 & 22,200 & 1,828 \\
\hline \multirow{2}{*}{$\begin{array}{l}\text { TRATAM. } \\
\mathrm{mM} \text { de Al }\end{array}$} & \multicolumn{4}{|c|}{ Diâmetro do Caule (cm) } & \multicolumn{3}{|c|}{ Comprimento da raiz $(\mathrm{m})$} & \multicolumn{3}{|c|}{ Peso/nº Folhas } \\
\hline & \multicolumn{2}{|l|}{ Média } & \multicolumn{2}{|c|}{ Desvio.padrão. } & Média & \multicolumn{2}{|c|}{ Desvio.padrão } & Médi & & esvio.padrão \\
\hline 0 & \multicolumn{3}{|c|}{0,318} & 0,041 & 118,706 & & 100,148 & & 0,113 & 0,025 \\
\hline 0,5 & \multicolumn{3}{|c|}{0,297} & 0,040 & 93,247 & & 26,265 & & 0,108 & 0,030 \\
\hline 1,0 & \multicolumn{3}{|c|}{0,325} & 0,036 & 117,563 & & 55,386 & & 0,138 & 0,030 \\
\hline 2,0 & \multicolumn{3}{|c|}{0,332} & 0,048 & 127,906 & & 83,223 & & 0,142 & 0,030 \\
\hline 4,0 & &, 335 & & 0,036 & 128,589 & & 32,801 & & 0,154 & 0,017 \\
\hline 6,0 & & ,398 & & 0,019 & 166,875 & & 41,238 & & 0,181 & 0,024 \\
\hline
\end{tabular}

Tabela 3: Teores de $\mathrm{Al}(\mathrm{mM})$ medidos ao final do experimento na solução hidropônica

Table 3: $\quad$ Al levels (mM) measured at the end of the experiment in the hydroponic solution

\begin{tabular}{|c|c|c|c|c|c|c|c|c|c|c|c|}
\hline Tratam & Caixas & $\begin{array}{l}\mathrm{Al}^{(1)} \\
\text { mom. }\end{array}$ & $\%^{(6)}$ & $\mathrm{Al}^{(2)}$ & $\%^{(6)}$ & $\begin{array}{c}\text { Al total } \\
\text { dig.(3) }^{\text {(3) }}\end{array}$ & $\%^{(6)}$ & $\begin{array}{c}\mathrm{Al} \text { dig }-\mathrm{Al} \\
\text { mon. }^{(4)}\end{array}$ & $\%^{(6)}$ & $\begin{array}{c}\mathrm{Al} \text { dig - } \\
\text { Al total }^{(5)}\end{array}$ & $\%^{(6)}$ \\
\hline 0 & $\mathrm{~A}$ & 0,03 & 0 & 0,01 & 0 & 0,00 & 0 & $-0,03$ & 0 & $-0,01$ & U \\
\hline 0 & B & 0,01 & 0 & 0,01 & 0 & 0,00 & 0 & $-0,01$ & 0 & $-0,01$ & 0 \\
\hline 0,5 & A & 0,08 & 9 & 0,09 & 1 & 0,44 & 89 & 0,36 & 72 & 0,36 & 71 \\
\hline 0,5 & B & 0,15 & 21 & 0,11 & 5 & 0,54 & 107 & 0,39 & 77 & 0,43 & 86 \\
\hline 1 & A & 0,23 & 30 & 0,20 & 1 & 0,98 & 98 & 0,75 & 75 & 0,79 & 79 \\
\hline 1 & B & 0,31 & 33 & 0,21 & 3 & 1,04 & 104 & 0,72 & 72 & 0,83 & 83 \\
\hline 2 & A & 0,54 & 21 & 0,37 & 14 & 1,85 & 93 & 1,31 & 66 & 1,48 & 74 \\
\hline 2 & B & 0,53 & 27 & 0,38 & 13 & 1,89 & 94 & 1,36 & 68 & 1,51 & 76 \\
\hline 4 & A & 1,26 & 30 & 0,76 & 11 & 3,81 & 95 & 2,56 & 64 & 3,05 & 76 \\
\hline 4 & B & 1,31 & 34 & 0,61 & 15 & 3,04 & 76 & 1,72 & 43 & 2,43 & 61 \\
\hline 6 & A & 1,40 & 22 & 0,90 & 14 & 4,48 & 75 & 3,08 & 51 & 3,59 & 60 \\
\hline 6 & B & 1,37 & 20 & 1,13 & 11 & 5,65 & 94 & 4,28 & 71 & 4,52 & 75 \\
\hline
\end{tabular}

(1) Al monom.- Al momomérico, medido por método colorimétrico

(2) Al total - medido em espectrofotômetro UV/VIS

(3) Al total dig.- Al total digerido medido em espectrofotômetro UV/VIS após incineração da amostra em mufla e diluição em $\mathrm{HCl}$.

(4) $\mathrm{Al}$ dig - $\mathrm{Al}$ mon.- $\mathrm{Al}$ total digerido menos o $\mathrm{Al}$ monomérico

(5) $\mathrm{Al}$ dig - Al total - $\mathrm{Al}$ total digerido menos $\mathrm{Al}$ total

(6) \% em relação aos teores de $\mathrm{Al}$ dos tratamentos

Na tabela 3 constam os resultados das análises do Al na solução nutritiva a $\mathrm{pH} 4,0$. Os valores do Al monomérico alcançaram valores que variaram em termos de percentagem em relação aos tratamentos de 20 a 35\%. Já o Al medido diretamente da solução nutritiva apresentou percentagens mais baixas variando de 1 a $15 \%$. A diferença entre o Al após e antes da combustão apresentou valores maiores que $60 \%$ em relação ao total de $\mathrm{Al}$ acrescentado na solução, e a diferença com Al monomérico ocorreu em uma faixa de 50 a $75 \%$. Todas estas medidas estão altamente correlacionadas, a maior parte com coeficientes de determinação acima de 0,96 (tab. 4). 

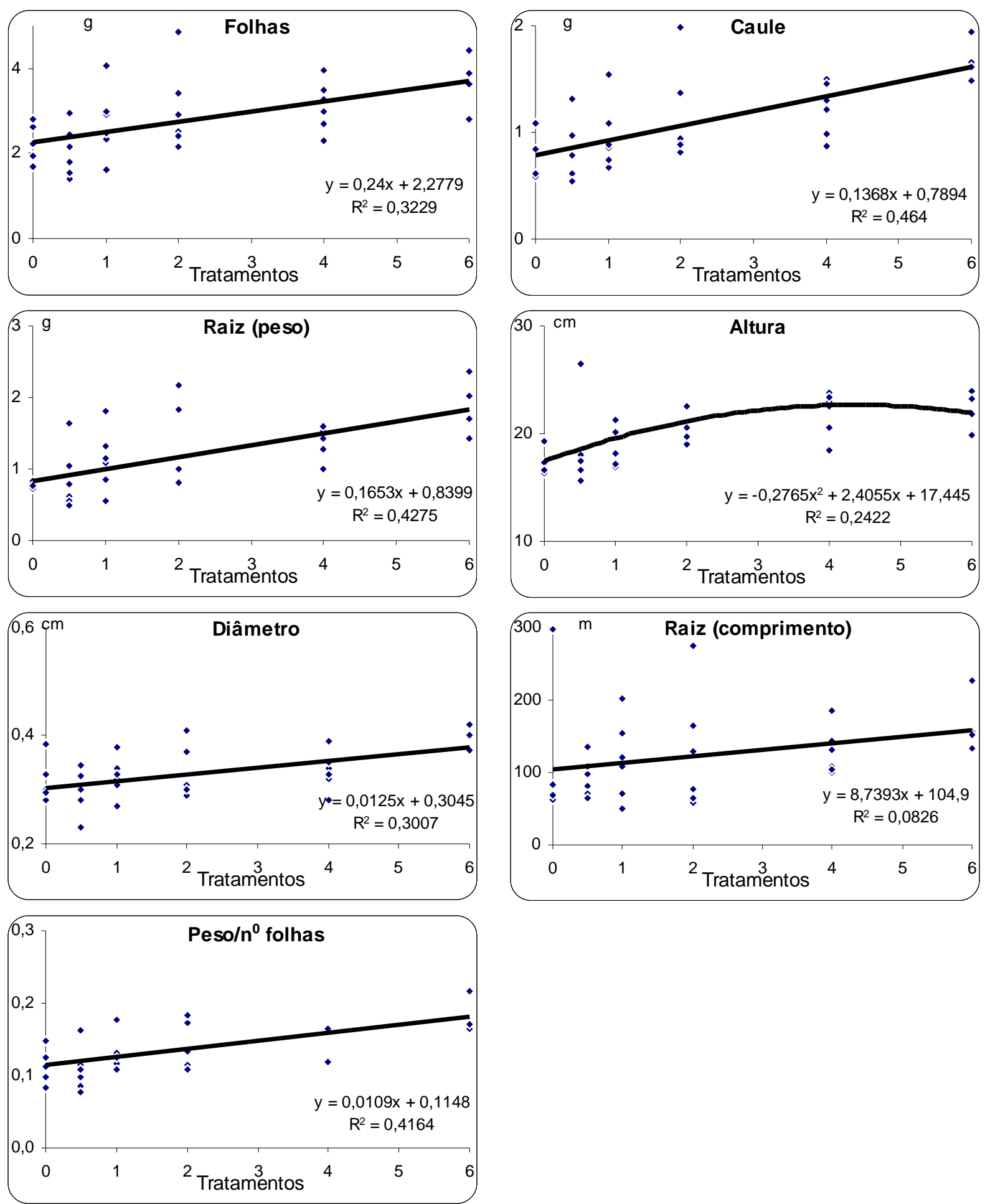

Figura 1: Ajuste de curvas de regressão de medidas realizadas em Tapirira guianensis, frente a níveis crescentes de $\mathrm{Al}(\mathrm{mM})$

Figure 1: Adjusted regression functions to increasing levels of se Al (mM) for al measured variables on Tapirira guianensis 
Tabela 4: Índices de correlação entre as diversas formas de Al na solução nutritiva hidropônica

Table 4: Correlation index among Al in several forms in the hydroponic solution

\begin{tabular}{l|c|c|c|c|c|c}
\hline & Tratamen. & Al monom. & Al tot. diger. & Al total & Al dig-Al mon. & Al dig.-Al total \\
\hline Tratamentos & 1,00 & & & & \\
Al monomér. & $0,97^{* *}$ & 1,00 & & & \\
Al total diger. & $0,99^{* *}$ & $0,96^{* *}$ & 1,00 & & \\
Al total & $0,99^{* *}$ & $0,96^{* *}$ & $1,00^{* *}$ & 1,00 & \\
Al dig.-Al mon. & $0,97 * *$ & $0,92^{* *}$ & $0,99 * *$ & $0,99 * *$ & 1,00 & 1,00 \\
Al dig.-Al total & $0,99 * *$ & $0,96^{* *}$ & $1,09^{* *}$ & $1,00^{* *}$ & $0,99 * *$ &
\end{tabular}

* significativo a $90 \%$ de probabilidade

** significativo a $95 \%$ de probabilidade

Ao se analisar o Al na solução nutritiva por absorção atômica antes e após a queima por combustão, os teores de $\mathrm{Al}$ aumentaram consideravelmente, apresentando uma relação linear alta, com um coeficiente de determinação de 0,99 (tab. 4).

As espécies de $\mathrm{Al}$ na solução, provavelmente possuíam uma quantidade maior de $\mathrm{Al}$ ligado a complexos orgânicos em relação ao $\mathrm{Al}$ inorgânico em todos os tratamentos com $\mathrm{Al}$, o que pode significar uma alta atividade de exsudação de complexos orgânicos pela raiz, propiciando em grande parte a neutralização de Al tóxico na solução.

Em relação ao Al inorgânico provavelmente este esteja na forma predominante de $\mathrm{Al}^{3+}$, visto que em $\mathrm{pH} \mathrm{4}$, as formas de hidróxidos de $\mathrm{Al}$, que são as mais tóxicas, estão presentes em pequena quantidade.

Em solução nutritiva, baixos níveis de $\mathrm{Al}(\mu \mathrm{M})$ causa redução no crescimento da raiz em variedades de trigo e milho sensíveis ao Al (Pellet et al., 1995; 1996). Árvores de regiões temperadas (Picea abies) são tolerantes a concentrações acima de $0,3 \mathrm{mM}$ de $\mathrm{Al}$ antes de seu crescimento ser reduzido (Heim et al., 1999)..

T. guianensis exibe alta plasticidade em termos ambientais, ocorrendo em ambientes variados desde a América Central ao sul do Brasil, inclusive em solos com altos teores de $\mathrm{Al}$, como os do Cerrado, embora submetida a altas doses de $\mathrm{Al}(6 \mathrm{mM})$ ainda apresenta desenvolvimento crescente (fig. 1).

Dentre os possíveis mecanismos discutidos na literatura que envolve a tolerância ao Al de T. guianensis cita-se, o de exclusão do Al no apoplasma (espaço intracelular e da parede celular), através da excreção de substâncias orgânicas que ligando-se a este formam complexos estáveis, que apresentam menos fitotoxidade que os íons livres $\mathrm{Al}^{3+}$ (Hue et al., 1986), e os relacionados a excreção de ácidos orgânicos na solução nutritiva, comuns em plantas agrícolas (Delhaize et al., 1993; Pellet et al., 1995; Zheng et al., 1998). Em espécies arbóreas de pinheiros, Smith (1969), constatou em solução nutritiva a exsudação de ácidos orgânicos.

Neste experimento foram encontrados apenas traços de ácidos orgânicos na solução nutritiva. Segundo Marschner (1991), é difícil constatar a presença de ácidos orgânicos em solução nutritiva, não só pela baixa produção de exsudados, mas também pela rápida degradação por microorganismos e pela diluição na solução. Heim et al. (1999), em Picea abies, em solução nutritiva, atribui a tolerância ao $\mathrm{Al}$ a imobilização no apoplasto e não pela exsudação de substâncias orgânicas. Ao revisar este tema, salientou que existe pouca informação relacionada a este mecanismo em espécies florestais.

Visualmente foi constatada a presença de uma mucilagem no entorno da raiz. Horst et al. (1982), encontrou uma mucilagem ao redor da raiz de "cowpea" composta por polissacarídeos e ácidos poliurônicos secretados pelas células da raiz, atribuindo como mecanismo de tolerância ao Al. Esta mucilagem é altamente resistente a degradação microbiológica e pode ter um efeito inibidor da toxidez com Al, mas necessita ser melhor estudada (Marschner, 1991).

Os possíveis mecanismos que inibem a toxidez do $\mathrm{Al}$ em Tapirira guianensis estão relacionados à excreção de substâncias orgânicas que quelam o Al tornando-o menos tóxico no meio, ou relacionados ao acúmulo na 
raiz não permitindo que parcialmente este não seja transportado para a parte aérea em quantidades que possam torná-lo tóxico, ou ainda, que fosse transportado já complexado com alguma substância ou elemento que neutraliza sua toxidez.

\section{Teor e conteúdo de nutrientes na planta}

A figura 2 ilustra o comportamento dos teores de nutrientes e $\mathrm{Al}$, nas diferentes partes de $T$. guianensis, submetida a tratamentos com níveis crescentes de $\mathrm{Al}$, através do ajuste de curvas de regressão. Todos os elementos analisados apresentaram diferenças significativas, ao nível de $1 \%$ de probabilidade, entre tratamentos. Todos os nutrientes tendem a diminuir com a aplicação do Al. Os teores de Al são crescentes nas folhas, caule e raízes.

Os teores de $\mathrm{Al}$ na testemunha são maiores na raiz, seguido das folhas e posteriormente no caule (fig. 2). Quando dos tratamentos com $\mathrm{Al}$, o teor na raiz aumenta mais de 20 vezes no primeiro tratamento $(0,5$ $\mathrm{mM})$ e mais de 50 vezes no último $(6 \mathrm{mM})$. No caule este aumento é de cerca de 9 vezes, e na folha, apenas de uma vez e meia. O aumento é gradativo a partir dos tratamentos com $\mathrm{Al}$, sendo significativamente maior no tratamento com $6 \mathrm{mM}$, principalmente na folha e na raiz.

A correlação entre os teores de nutrientes foi significativa para boa parte dos nutrientes. Só os índices significativamente correlacionados com o $\mathrm{Al}$ foram negativos.

Reunindo as variáveis relacionadas através da análise fatorial (fig. 3). Nas três frações da planta, obteve-se 3 fatores. Nas folhas, estes explicam $77,47 \%$ da variância total, no caule, $80,27 \%$, e na raiz, $83,14 \%$. Esta avaliação permite agrupar os elementos que tiveram comportamento similar frente aos níveis crescentes de $\mathrm{Al}$ na solução nutritiva.

$\mathrm{Na}$ raiz o primeiro fator foi composto pelos nutrientes $\mathrm{N}, \mathrm{Fe}, \mathrm{Cu}, \mathrm{Zn}$ e pelo $\mathrm{Al}$ (fig. 3). Com exceção do $\mathrm{Al}$, estes elementos tiveram uma diminuição em seus teores, perante as doses crescentes de $\mathrm{Al}$ e um posterior aumento no tratamento com $6 \mathrm{mM}$ (fig. 2).

O segundo fator foi constituído por $\mathrm{P}$, $\mathrm{Ca}, \mathrm{Mn}$ e $\mathrm{Mg}$ (fig. 3), com uma tendência de diminuição e uma posterior estabilização da curva. O P, apresentou um decréscimo drástico em seus teores (fig. 2).

$\mathrm{O} \mathrm{K}$ ficou isolado na análise fatorial (fig. 3), o que representa uma resposta diferenciada perante os tratamentos com $\mathrm{Al}$ na raiz.

$\mathrm{Na}$ fração caule, os elementos $\mathrm{P}, \mathrm{N}$, $\mathrm{Cu}, \mathrm{Ca}$ e $\mathrm{Mg}$, foram agrupados no fator 1 , com uma tendência de decréscimo e posterior aumento (fig. 2).

$\mathrm{O}$ outro fator, foi composto por Fe, $\mathrm{Mn}, \mathrm{Zn}$ e $\mathrm{K}$, sempre tendendo a uma diminuição perante os tratamentos (fig. 2). $\mathrm{O} \mathrm{Al}$ ficou isolado em um fator (fig. 3), devido ao aumento intenso ocorrido (fig. 2).

$\mathrm{Na}$ fração folhas, os teores de $\mathrm{P}, \mathrm{K}$, Mn e N, participam de um mesmo fator (fig. 3), caracterizado por um decréscimo durante os tratamentos com $\mathrm{Al}$ e um leve aumento no tratamento com dose mais elevada $(6 \mathrm{mM})$, depois do ajuste da curva da regressão. Para o P ocorreu um decréscimo nos teores mais drástico, como já havia ocorrido na raiz e no caule (fig. 2).

Os elementos $\mathrm{Mg}$, Al agruparam-se em outro fator, devido a uma tendência de diminuição (Mg) e aumento (Al) mais acentuado (fig. 2). O P, também apresentou pesos elevados neste fator apresentando um auto índice de correlação com o Mg.

$\mathrm{O} \mathrm{Ca}$ apresentou uma alta variância específica, não enquadrando-se em nenhum fator (fig. 3), embora tenha apresentado índices de correlação significativos com os demais elementos.

Os elementos $\mathrm{Fe}, \mathrm{Cu}$ e $\mathrm{Zn}$, apresentaram comportamento similar, com uma tendência de aumento até o tratamento com 1 $\mathrm{mM}$ de $\mathrm{Al}$ e uma posterior diminuição, no ajuste das curvas de regressão tiveram tendência a diminuição mas pouco significativa (fig. 2). 
Efeito do alumínio no desenvolvimento...

Figura 2: Ajuste de curvas de regressão entre os teores de nutrientes medidos nas frações de tapirira guianensis e os tratramentos com níveis crescentes de al (mm).

Figure 2: Regression curves adjustment among nutrients content measured in the fractions of tapirira guianensis and treatments with al increasing levels ( $\mathrm{mm}$ ).
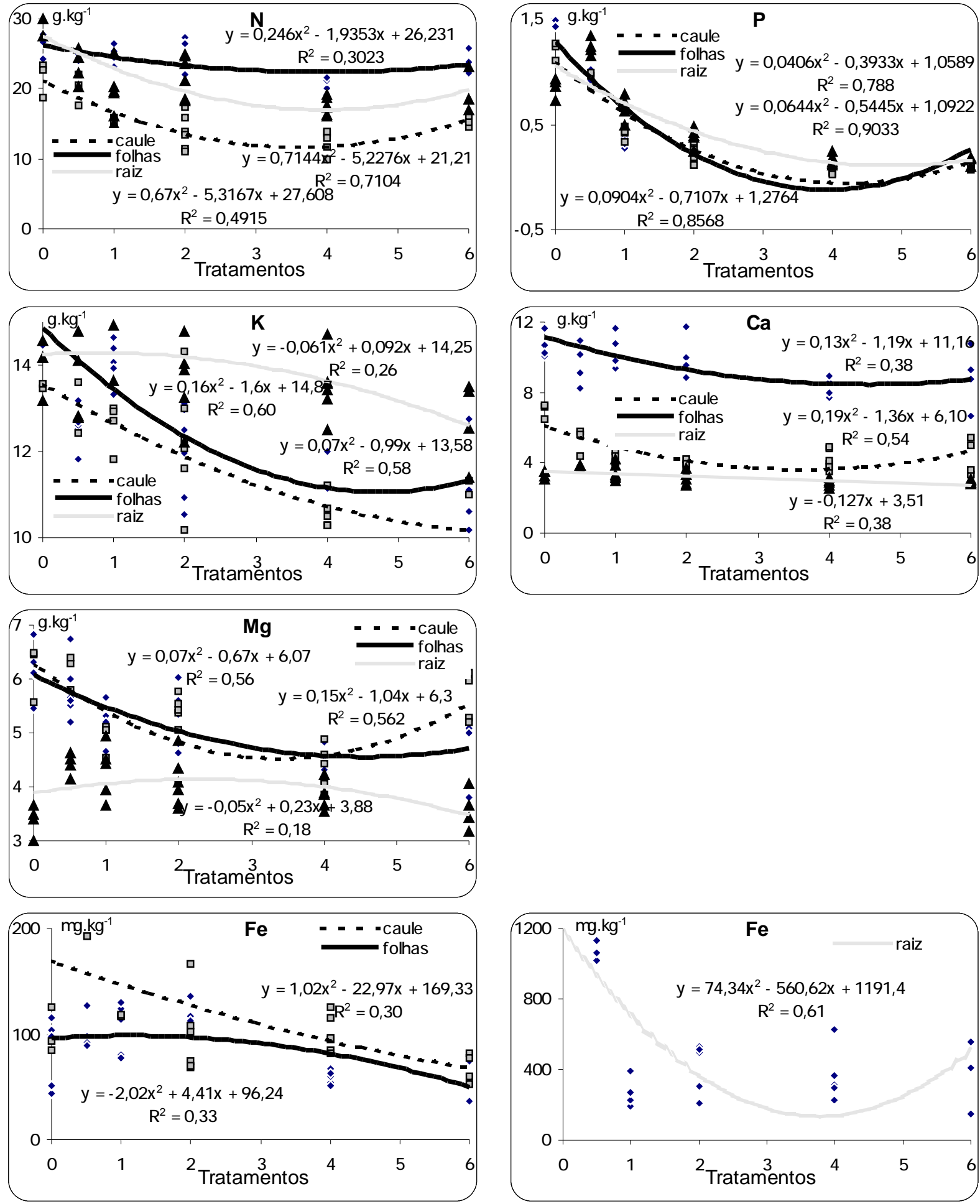

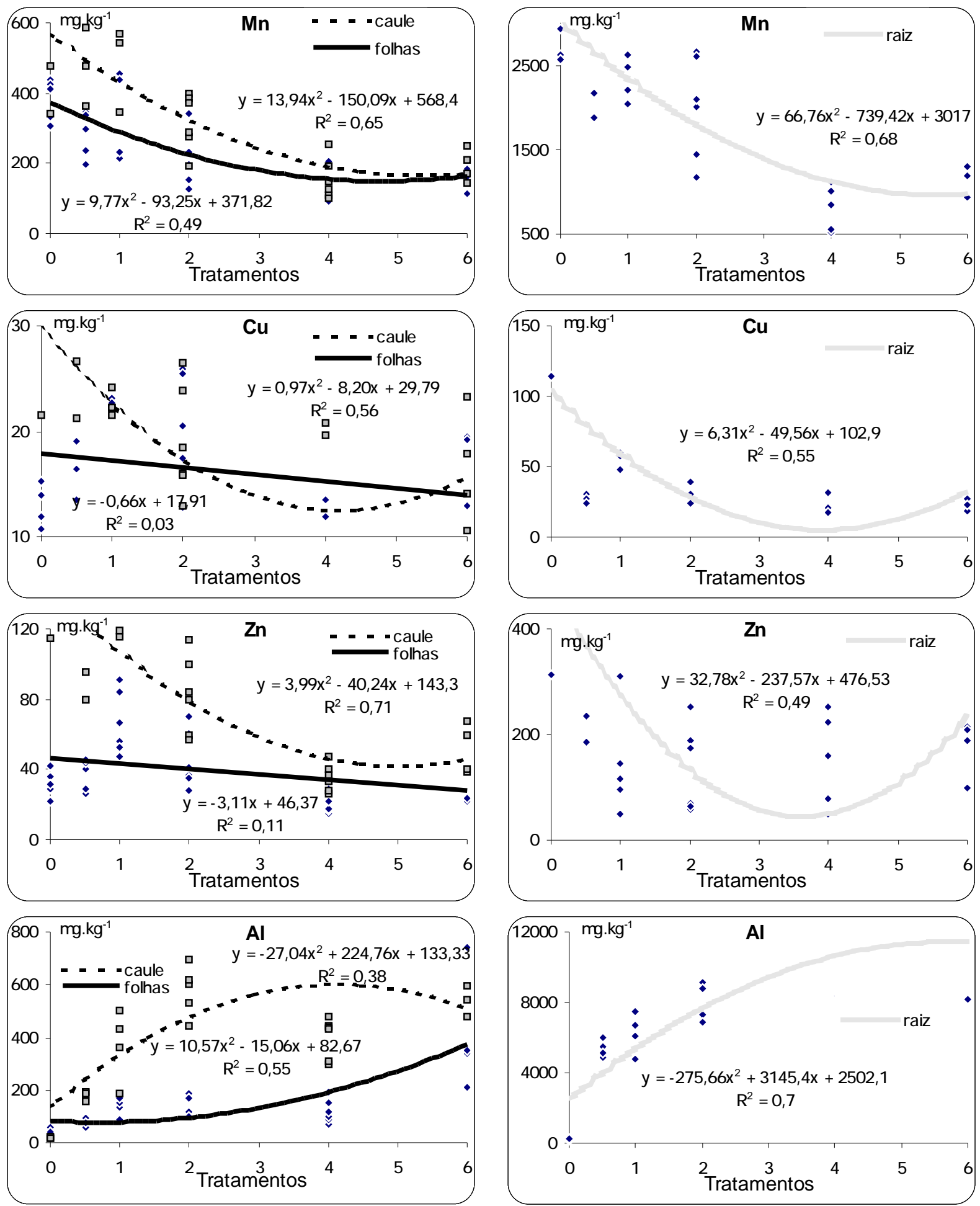
Efeito do alumínio no desenvolvimento...

Figura 3: Representação gráfica dos pesos fatoriais da variável teor de nutrientes das frações de tapirira guianensis, após rotação varimax.

Figure 3: Graphic representation from factorial weights of the nutrients variable content of the fractions of tapirira guianensis, after varimax rotation.
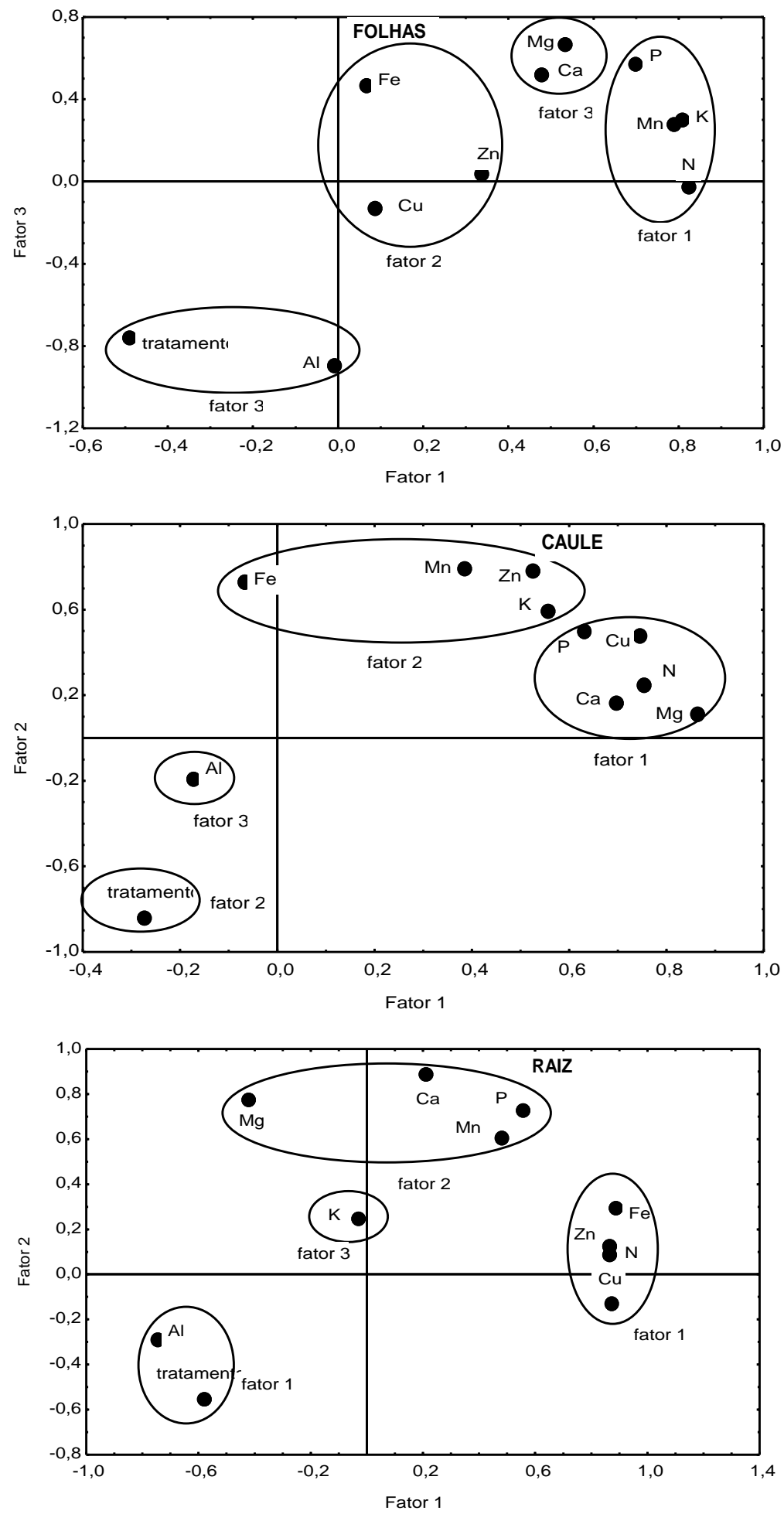
Através desta avaliação constatam-se 3 tendências representativas do comportamento dos nutrientes frente à níveis crescentes de $\mathrm{Al}$. A primeira delas ocorre um decréscimo nos teores e uma tendência de aumento no ultimo tratamento $(6 \mathrm{mM})$, provavelmente representando uma reação mais pronunciada da planta na dose mais elevada, refletindo no aumento dos teores. Na segunda, não ocorre esta resposta no ultimo tratamento, desta forma, os elementos com este comportamento foram os que apresentaram os índices de correlação mais elevados, tanto em termos de teores de $\mathrm{Al}$ na solução nutritiva, quanto em relação aos parâmetros biométricos da planta. A terceira tendência indica um comportamento variável frente aos níveis crescentes de $\mathrm{Al}$.

Em virtude do aumento da biomassa da planta, quando dos tratamentos com $\mathrm{Al}$, os teores tendem a diminuir ("efeito de diluição"), mascarando as quantidades absorvidas dos elementos pela planta. Entretanto, ao multiplicar-se os teores de nutrientes pela biomassa da planta, obtêm-se o conteúdo de nutrientes na planta, ou as quantidades de nutrientes absorvidas pela mesma.

Em relação ao conteúdo de nutrientes, a análise de variância apontou diferenças significativas (nível de significância de 1 e 5\%) entre tratamentos dos seguintes elementos na fração folhas: $\mathrm{N}, \mathrm{P}, \mathrm{Fe}, \mathrm{Cu}, \mathrm{Zn}$ e $\mathrm{Al}$; na fração caule, $\mathrm{N}, \mathrm{P}, \mathrm{Ca}, \mathrm{Mg}, \mathrm{Fe}, \mathrm{Mn}, \mathrm{Cu}, \mathrm{Zn}$, e Al, e na fração raiz, $\mathrm{P}, \mathrm{Ca}, \mathrm{Mg}, \mathrm{Fe}, \mathrm{Mn}, \mathrm{Cu}, \mathrm{Zn}$ e Al.

Os elementos que tiveram um aumento significativo ou tendência de aumento em suas quantidades foram $\mathrm{N}, \mathrm{K}, \mathrm{Ca}, \mathrm{Mg}$ e $\mathrm{Al}$. Os micronutrientes tiveram um padrão irregular nos diferentes tratamentos, e o $\mathrm{P}$, diminuiu significativamente (fig. 4).

Os índices de correlação entre o conteúdo de nutrientes nas folhas, foram significativos entre quase todos os nutrientes, com exceção do $\mathrm{P}$, que correlacionou-se somente com o Mn e Al.

Agrupando-se as variáveis relacionadas através da análise fatorial, foram extraídos 3 fatores. Nas folhas estes explicaram $87,40 \%$ da variância total, no caule $81,84 \%$, e na raiz $85,41 \%$. A figura 5 possibilita a visualização dos agrupamentos dos nutrientes nos fatores, tendo como eixos o fator 1 e 2 .
$\mathrm{Na}$ fração raiz, foram agrupados $\mathrm{Fe}$, $\mathrm{Zn} \mathrm{e} \mathrm{Cu}$ (fig. 5). Observa-se pelo ajustes das curvas de regressão um decréscimo e posterior aumento nas quantidades absorvidas durante os tratamentos com $\mathrm{Al}$ (fig. 4).

Quanto ao N, K, Ca, e Mg estes foram agrupados em um outro fator, representando os nutrientes que foram absorvidos em maiores quantidades, o que era de se esperar para os macronutrientes. Da mesma forma o $\mathrm{Al}$, que também faz parte deste fator, mas com um grande acumulo ocorrido na raiz.

$\mathrm{O} \mathrm{P}$ e Mn participaram de um outro fator, pela tendência que apresentam em termos de diminuição das quantidades absorvidas (fig. 4).

$\mathrm{Na}$ fração caule, também os macronutrientes $(\mathrm{N}, \mathrm{K}, \mathrm{Ca}, \mathrm{Mg})$ e $\mathrm{Al}$, foram agrupados no fator 1 , representando os elementos que foram absorvidos em maiores quantidades (fig. 5). O fator 2 agrupou, $\mathrm{P}, \mathrm{Mn}$, $\mathrm{Cu}$ e $\mathrm{Zn}$, devido a uma tendência de estabilização na absorção destes elementos (fig. 4).

O Fe no caule, permaneceu isolado, não correlacionando-se com nenhum dos outros elementos (fig. 5).

$\mathrm{Na}$ fração folhas, $\mathrm{Al}$ e $\mathrm{P}$, ficaram agrupados (fig. 5). O primeiro por uma uma absorção mais elevada que os demais elementos, e o segundo, por uma diminuição mais acentuada em sua absorção, sendo o único nutriente que tanto em termos de teores, quanto de conteúdo, diminuiu seus valores perante os tratamentos com $\mathrm{Al}$ (fig. 4). Uma das explicações plausíveis seria a falta de micorrizas nas plantas em solução nutritiva, característica importante para absorção deste nutriente.

Da mesma forma que na raiz e no caule, as quantidades de $\mathrm{N}, \mathrm{Ca}, \mathrm{Mg}$ e $\mathrm{K}$, reuniram-se no fator 1 (fig. 4), todos com tendência de aumento nas quantidades absorvidas.

Os micronutrientes reuniram-se no fator 3 (fig. 5), com a curva da regressão tendendo a estabilização em relação ao ajuste realizado entre as quantidades absorvidas e as doses crescentes de $\mathrm{Al}$ da solução (fig. 4). 
Efeito do alumínio no desenvolvimento...

Figura 4: Ajuste de curvas de regressão entre o conteúdo de nutrientes medidos nas frações de tapirira guianensis e os tratramentos com níveis crescentes de al (mm).

Figure 4: Regression curves adjustment among nutrients content measured in the fractions of tapirira guianensis and treatments with al increasing levels ( $\mathrm{mm})$.
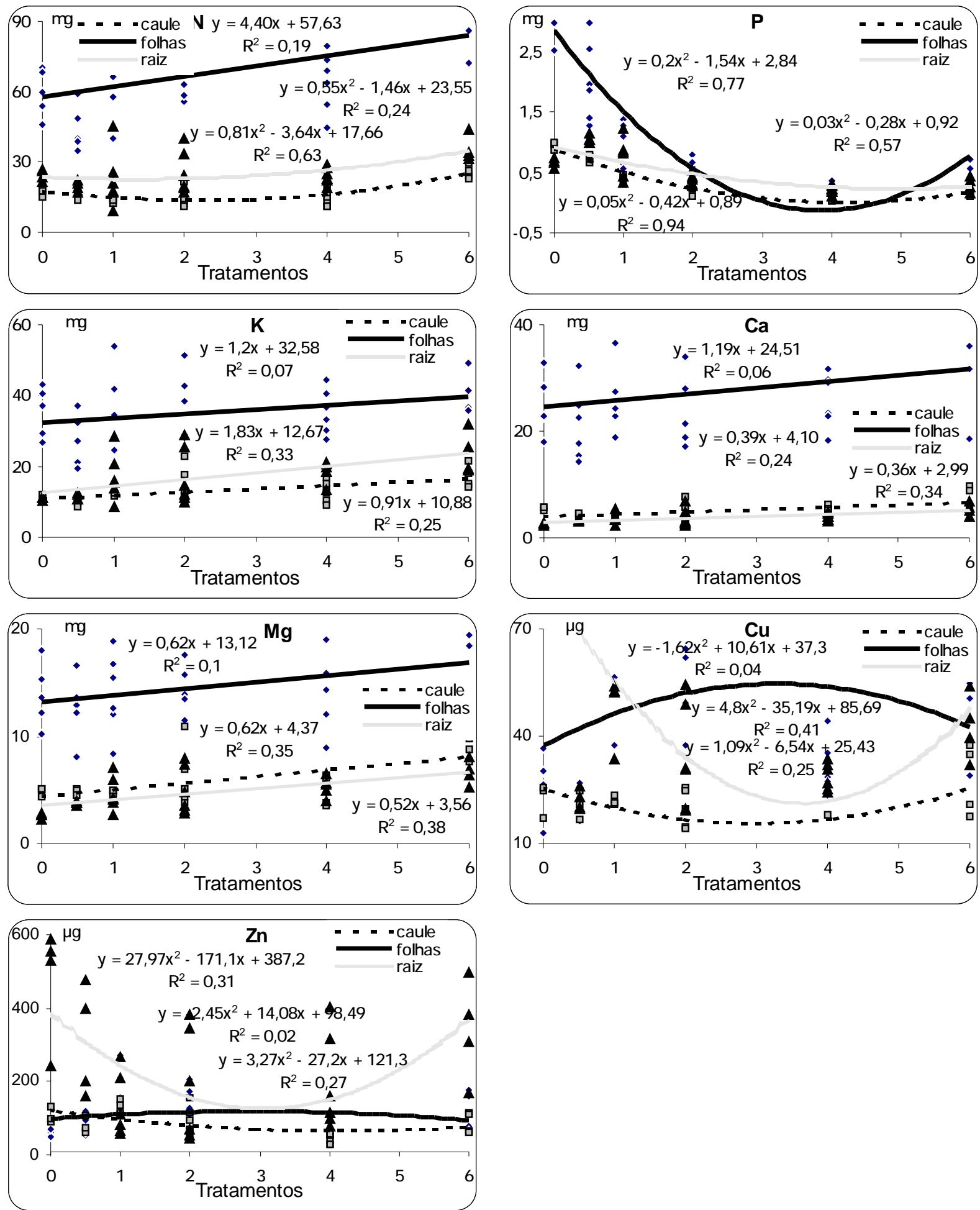

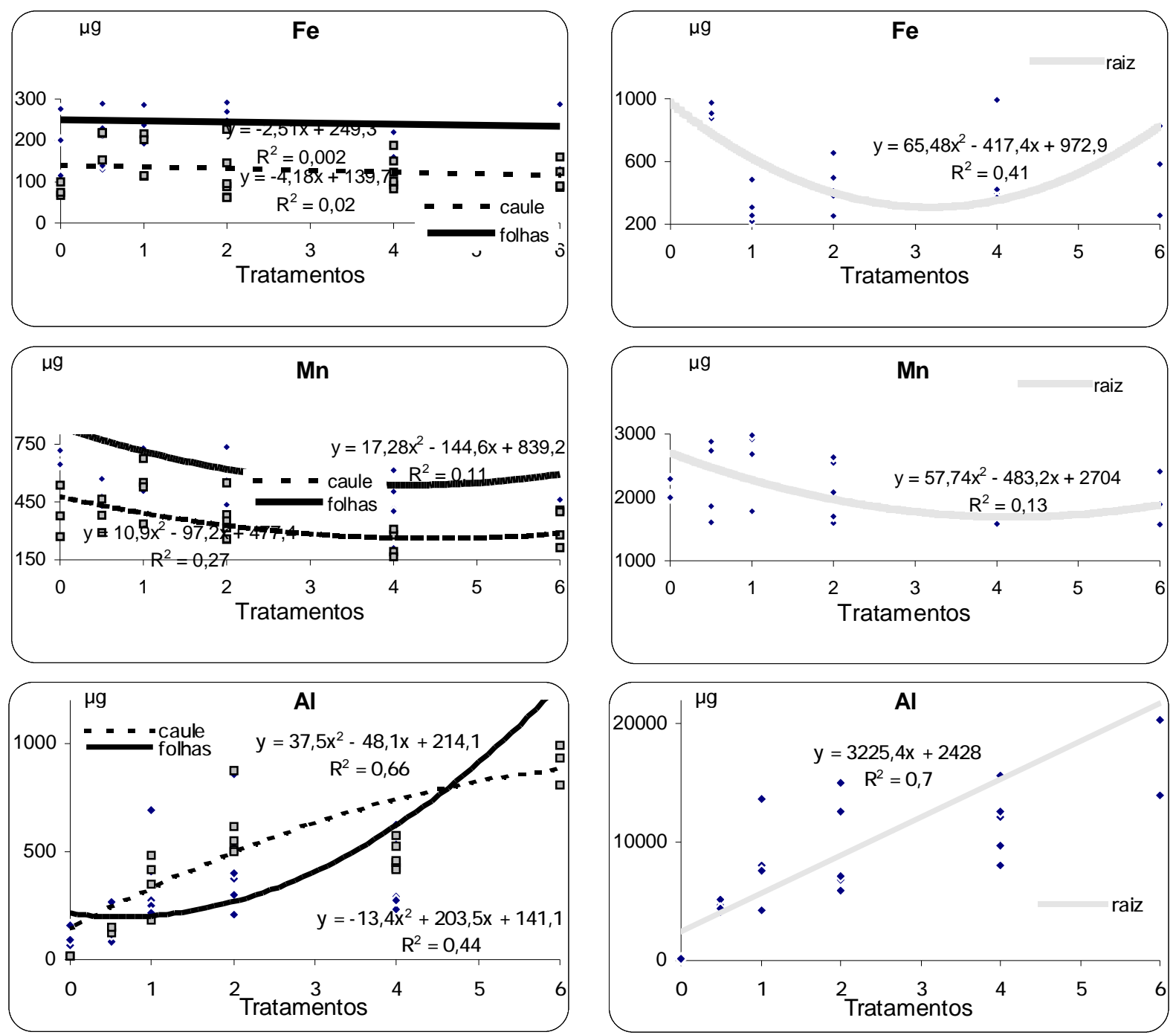
Efeito do alumínio no desenvolvimento...

Figura 5: Representação gráfica dos pesos fatoriais da variável conteúdo de nutrientes das frações de tapirira guianensis, após rotação varimax.

Figure 5: Graphic representation from factorial weights of the nutrients variable content of the fractions of tapirira guianensis, after varimax rotation.
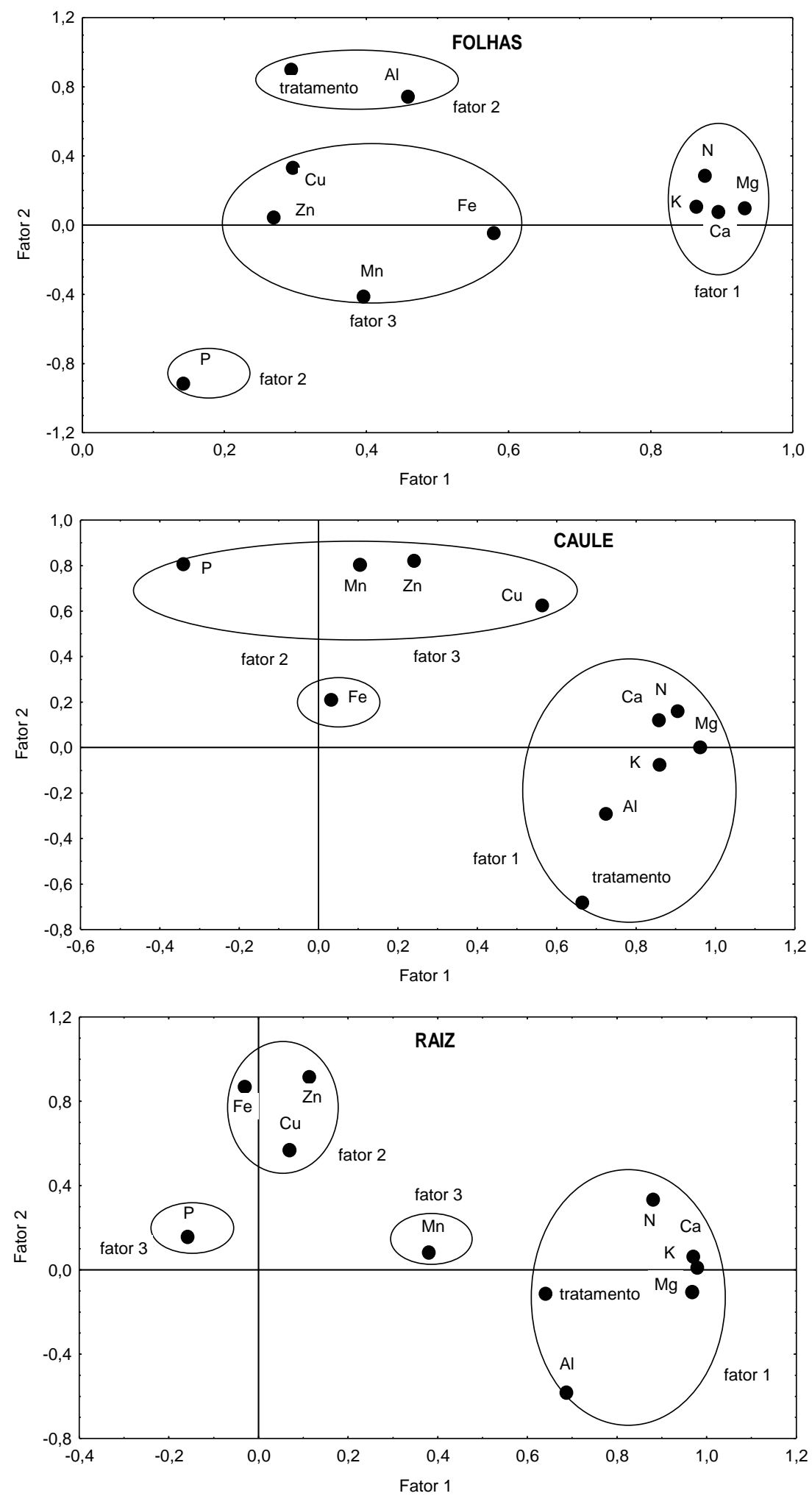
Britez, R. M. de; et al 
OSAKI, M.; WATANABE, T.; TADANO, T. Beneficial effect of aluminum on growth of plants adapted to low $\mathrm{pH}$ soils. Soil-Scienceand-Plant-Nutrition.43: 3, 551-563,1997.

PAVAN, M.A. \& BINGHAM F. T. 1982. Toxicity of aluminum to coffee seedlinfs grown in nutrient solution. Soil Sci. Soc. Am. J. 46:993-997.

PELLET, D. M.; GRUNES D. L.; KOCHIAN L. V. 1995. Organic acid exudation as an aluminum-tolerance mechanism in maize (Zea mays L.). Planta, 196:788-795.

PELLET, D. M.; PAPERNIK, L. A.; KOCHIAN, V. 1996. Multiple aluminumresistance mechanisms in wheat. Rles of root apical phosphate and malate exudation. Plant Physiology, 112:591-597.

SMITH, W. 1969. Release of organic materials from the rorts of tree seedlings. Forest Science, v.15(2): 138-143.
SPOSITO, G. 1996. The environmental chemistry of aluminum. Lewis Publishers. 464p.

TENNANT, D. 1975. A test of modified line intersect method of estimating root length. Journal of Ecology, 64:995-1001.

UEXKÜLL H.R. VON \& MUTERT E. 1995. Global extent, development and economic impact of acid soils. Plant and Soil, 171:105112.

WHEELER D.M.; EDMEADES, D.C.; CHRISTIE , R. A. 1993. Effect of aluminium on the growth of 34 plant species: A summary of results obtained in low ionic stregth solution culture. In: Genetic aspects of plant mineral nutrition. P.J. Randall et al (eds) p. 75-80.

ZHENG, S. J.; MA, J. F.; MATSUMOTO, H. 1998. Continuos secretion of organic acids is related to aluminium resistance during relatively long term exposure to aluminium stress. Physiologia Plantarum, 103:209-214. 